\title{
Synthesis of $C_{3}$-symmetric star-shaped molecules containing $\alpha$-amino acids and dipeptides via Negishi coupling as a key step
}

Sambasivarao Kotha* and Saidulu Todeti

\author{
Full Research Paper \\ Address: \\ Department of Chemistry, Indian Institute of Technology-Bombay, \\ Powai, Mumbai-400076, India, Fax: +91(22)-2572 7152 \\ Email: \\ Sambasivarao Kotha* - srk@chem.iitb.ac.in \\ * Corresponding author \\ Keywords: \\ amino acids; cyclotrimerization; Negishi coupling; peptide
}

\author{
Beilstein J. Org. Chem. 2019, 15, 371-377. \\ doi:10.3762/bjoc. 15.33 \\ Received: 16 October 2018 \\ Accepted: 21 January 2019 \\ Published: 08 February 2019 \\ Associate Editor: D. Spring \\ (c) 2019 Kotha and Todeti; licensee Beilstein-Institut. \\ License and terms: see end of document.
}

\begin{abstract}
We demonstrate a new synthetic strategy toward star-shaped $C_{3}$-symmetric molecules containing $\alpha$-amino acid (AAA) derivatives and dipeptides. In this regard, trimerization and Negishi cross-coupling reactions are used as the key steps starting from readily available 4'-iodoacetophenone and L-serine. These $C_{3}$-symmetric molecules containing AAA moieties are useful to design new ligands suitable for asymmetric synthesis and peptide dendrimers.
\end{abstract}

\section{Introduction}

Optically active $C_{3}$-symmetric molecules are valuable synthons to design dendrimers, chiral ligands, polymers, and supramolecules [1-4]. In this regard, 1,3,5-triarylbenzene derivatives are helpful to design star-shaped $\alpha$-amino acids (AAAs) and they play an important role in biological systems. The tumor necrosis factor (TNF) superfamily belongs to trimeric ligands that form in the shape of $C_{3}$-symmetric molecules [5]. Trimeric proteins containing star-shaped compounds are also involved in the complex interactions between cells and pathogens, e.g., the human immunodeficiency virus (HIV-1) [6]. The HIV-1 envelope protein is present as a $C_{3}$-symmetric trimer on the viruses' surface [7], and the virus entry into the cell is mediated by its interactions with cellular receptors. To explore the structural and chemical nature of protein-protein interactions, synthetic peptides and unnatural AAAs [8-15] can be useful as molecular tools. Moreover, $C_{3}$-symmetric peptides are valuable in studying the molecular interactions involving proteins that are derived from trimers and synthetic access to such amino acids is vital. In this regard, new star-shaped $C_{3}$-symmetric molecules [16-26] have been used in photovoltaics [27,28], organic lightemitting diodes (OLEDs) [29,30], organic field-effect transistors (OFETs) [31,32] and electroluminescent devices [33]. To address these challenges, we [34] and others [35,36] have synthesized functionalized $C_{3}$-symmetric molecules containing amino acids and peptides.

The Negishi cross coupling [37,38] is a reliable synthetic method, which involves palladium or nickel-catalyzed coupling of organozinc reagents $[39,40]$ with various halo derivatives (e.g., aryl, vinyl, benzyl, or allyl) and has a broad scope to 
assemble diverse targets. This reaction was first reported in 1977, and it is an elegant and versatile method that allows the preparation of biaryls and olefins in good yields. To the best of our knowledge only a limited number of reports is available for the synthesis of $C_{3}$-symmetric peptides (Figure 1) $[8,41]$. To fill this gap, we have explored a new synthetic strategy to starshaped $C_{3}$-symmetric AAA derivatives and peptides by using trimerization and the Negishi cross coupling as key steps.

\section{Results and Discussion}

The required zinc insertion compound 7 was prepared from L-serine (3). Thus, commercially available L-serine (3) was treated with acetyl chloride in methanol to give methyl ester $\mathbf{4}$, which was subjected to $N$-Boc protection with di-tert-butyl dicarbonate $\left(\mathrm{Boc}_{2} \mathrm{O}\right)$ and triethylamine in tetrahydrofuran (THF) to obtain the $N$-Boc-serine methyl ester (5) in $93 \%$ yield [42]. Afterwards, the protected methyl ester 5 was subjected to iodination in the presence of iodine $\left(\mathrm{I}_{2}\right)$, triphenylphosphane
$\left(\mathrm{PPh}_{3}\right)$ and imidazole in $\mathrm{CH}_{2} \mathrm{Cl}_{2}$ at $0{ }^{\circ} \mathrm{C}$ to deliver the iodo derivative 6 in $63 \%$ yield $[43,44]$. Finally, the iodo compound 6 was treated with freshly activated $\mathrm{Zn}$ in DMF at room temperature to afford the zinc insertion product 7 (Scheme 1) [43].

With the organozinc compound 7 at hand we turned to the synthesis of the halide component for the attempted Negishi coupling. For this 4 -iodoacetophenone (8) was treated with silicon tetrachloride and ethanol $\left(\mathrm{SiCl}_{4} / \mathrm{EtOH}\right)$ at room temperature for $6 \mathrm{~h}$ to produce the iodonated trimerized product 9 in $71 \%$ yield (Scheme 2) $[45,46]$.

Then, the organozinc reagent 7 was coupled with triiodo derivative 9 in the presence of tetrakis(triphenylphosphane)palladium(0) $\left(\mathrm{Pd}\left(\mathrm{PPh}_{3}\right)_{4}\right)$ as catalyst to provide the Negishi coupling product $10(68 \%)$. Having the trimeric AAA derivative $\mathbf{1 0}$ in hand, it was treated with trifluoroacetic acid (TFA) in $\mathrm{CH}_{2} \mathrm{Cl}_{2}(1: 1)$ at room temperature for $1 \mathrm{~h}$ to deliver the Boc-

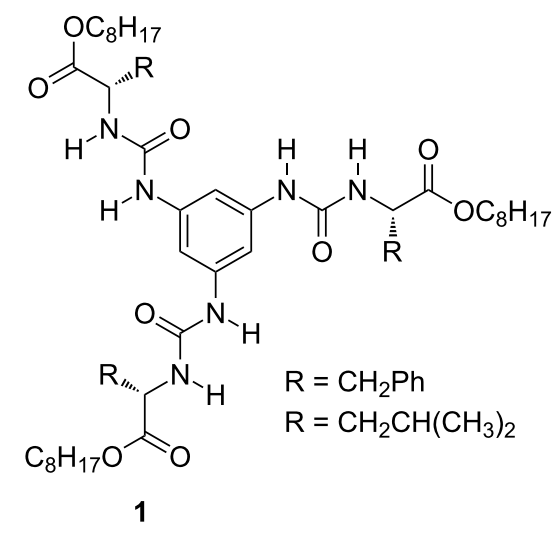<smiles>[X][X]CNC(=O)CONC(=O)c1cc(C(=O)NC[X])cc(C(=O)NCNC(=O)CON)c1</smiles>

$\mathrm{X}=-\left(\mathrm{CH}_{2}\right)_{2}-\mathrm{O}-\left(\mathrm{CH}_{2}\right)_{2}-\mathrm{O}-\left(\mathrm{CH}_{2}\right)_{2}-$

2

Figure 1: Exemplar $C_{3}$-symmetric peptide scaffolds reported in the literature.<smiles>NC(CO)C(=O)O</smiles>

3

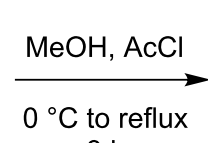

$3 \mathrm{~h}$<smiles>COC(=O)[C@H](N)Cl</smiles>

4

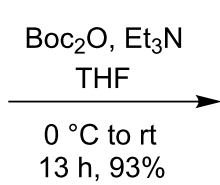

$13 \mathrm{~h}, 93 \%$<smiles>COC(=O)[C@H](CO)NC(=O)OCc1ccccc1</smiles>

$\mathrm{I}_{2}, \mathrm{PPh}_{3}$, imidazole DCM, $0{ }^{\circ} \mathrm{C}, 6 \mathrm{~h}, 63 \%$

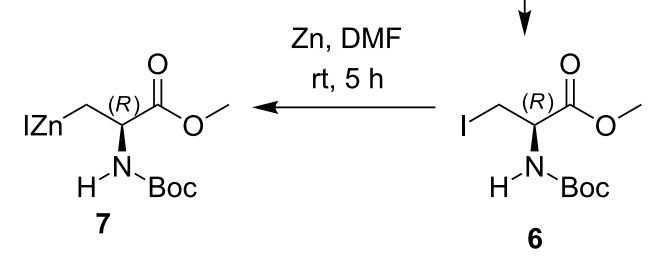




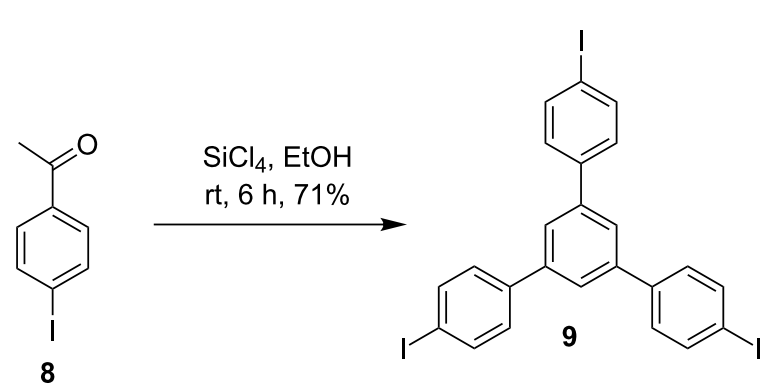

Scheme 2: Preparation of the trimerized product 9.

deprotected compound. Then, without further purification the deprotected product was directly treated with thiophene-2carboxylic acid in the presence of 2-(1H-benzotriazol-1-yl)1,1,3,3-tetramethyluronium hexafluorophosphate (HBTU) and $\mathrm{N}, \mathrm{N}$-diisopropylethylamine (DIPEA) in $\mathrm{CH}_{2} \mathrm{Cl}_{2}$ at room temperature for $5 \mathrm{~h}$ to give trimer $\mathbf{1 1}$ in $86 \%$ yield (Scheme 3 ).

In addition, different amino acids were incorporated in the starshaped molecule. In this regard, the Negishi cross-coupling product $\mathbf{1 0}$ was treated with TFA in $\mathrm{CH}_{2} \mathrm{Cl}_{2}$ at room temperature for $1 \mathrm{~h}$ to give the Boc-deprotection product, which was directly treated with Boc-Val-OH or Boc-Phe-OH in the presence of HBTU and DIPEA in $\mathrm{CH}_{2} \mathrm{Cl}_{2}$ at room temperature for $5 \mathrm{~h}$ to give trimeric derivatives $\mathbf{1 2}(73 \%)$ and $\mathbf{1 3}(81 \%)$, respectively. Further, trimer 12 was subjected to another Boc-deprotection to give the tris-amine $\mathbf{1 4}$ in $95 \%$ yield (Scheme 4 ).

\section{Conclusion}

We have demonstrated a simple synthetic strategy toward starshaped molecules containing unusual AAA units through cyclotrimerization and Negishi cross-coupling reaction as key steps under operationally simple reaction conditions. Here, we have used the readily available starting materials 4-iodoacetophenone (8) and L-serine (3). The $C_{3}$-symmetric building blocks prepared were coupled with different AAAs to produce the $C_{3}$-symmetric dipeptide trimers.

\section{Experimental \\ General procedure}

Commercially available starting materials were used without further purification. Analytical thin layer chromatography (TLC) was performed on $7.5 \times 2.5 \mathrm{~cm}$ glass plates coated with Acme's silica gel GF254 (containing 13\% calcium sulfate as binder) by using a suitable mixture of ethyl acetate and petro-<smiles>COC(=O)C(CC(C)C)NC(=O)OC(=O)c1ccc(-c2cc(-c3ccc(I)cc3)cc(-c3ccc(I)cc3)c2)cc1</smiles>

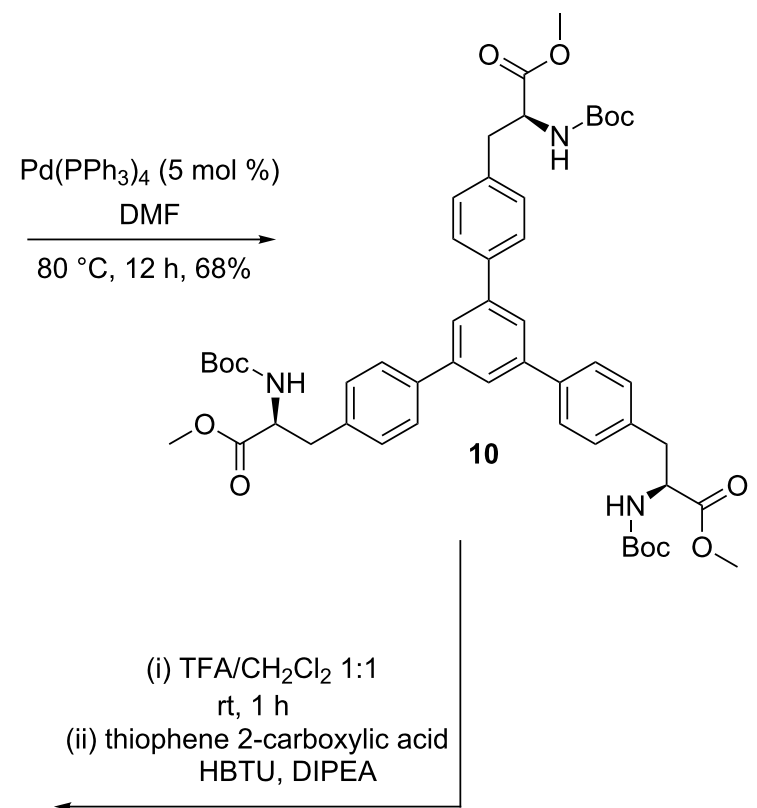<smiles>COC(=O)C(Cc1ccc(-c2cc(-c3ccc(CC(NC(=O)c4cccs4)C(=O)OC)cc3)cc(C(C)C)c2)cc1)NC(=O)c1cccs1</smiles>

$\mathrm{CH}_{2} \mathrm{Cl}_{2}$, rt, $5 \mathrm{~h}$,

two steps, $86 \%$ 


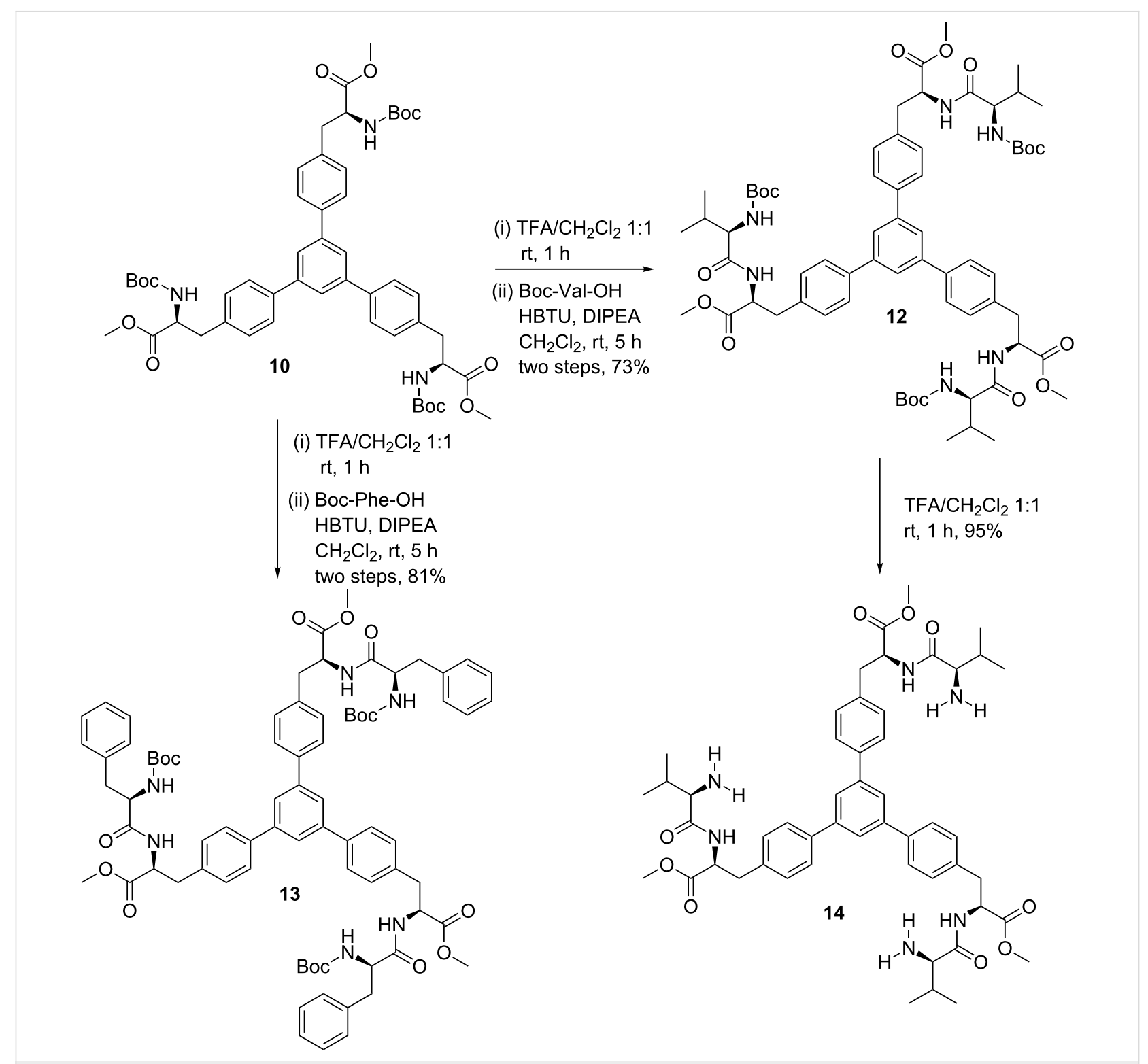

Scheme 4: Synthesis of $C_{3}$-symmetric trimers 12,13 and 14.

leum ether for development. The Negishi coupling was performed in oven-dried glassware under argon or nitrogen atmosphere and the transfer of moisture-sensitive materials was carried out in a glovebox by using standard syringe-septum techniques. All purchased solvents $\left(\mathrm{CH}_{2} \mathrm{Cl}_{2}\right.$, THF, acetonitrile, and DMF) were dried over calcium hydride $\left(\mathrm{CaH}_{2}\right)$ or sodium. Column chromatography was performed by using Acme's silica gel (100-200 mesh) with an appropriate mixture of ethyl acetate, petroleum ether methanol and dichloromethane. The coupling constants $(J)$ are given in hertz $(\mathrm{Hz})$ and chemical shifts are denoted in parts per million (ppm) downfield from internal standard, tetramethylsilane (TMS). The abbreviations, $\mathrm{s}, \mathrm{d}, \mathrm{t}, \mathrm{q}, \mathrm{m}$, and dd refer to singlet, doublet, triplet, quartet, multiplet, and doublet of doublets, respectively. Infrared (IR) spectra were recorded on a Nicolet Impact-400 FTIR spectrometer. Specific rotation experiments were measured at $589 \mathrm{~nm}$ (Na) and $25{ }^{\circ} \mathrm{C}$ ( $\mathrm{HPLC}, \mathrm{CHCl}_{3}$ stabilized with $0.7-1.0 \%$ ethanol). Proton nuclear magnetic resonance $\left({ }^{1} \mathrm{H}\right.$ NMR, $400 \mathrm{MHz}$ and $500 \mathrm{MHz}$ ) spectra and carbon nuclear magnetic resonance $\left({ }^{13} \mathrm{C} \mathrm{NMR}, 100 \mathrm{MHz}\right.$ and $\left.125 \mathrm{MHz}\right)$ spectra were recorded on a Bruker spectrometer. The high-resolution mass measurements were carried out by using electrospray ionization (ESI) spectrometer. Melting points were recorded on a Veego melting point apparatus.

\section{Negishi coupling product 10}

Zinc ( $\mathrm{Zn}$ ) dust was activated by using $3 \mathrm{M}$ aq $\mathrm{HCl}$, then filtered and washed with water (until neutral $\mathrm{pH}$ ) followed by acetone. 
Large particles were crushed until a fine powder was formed and transferred into a round-bottomed flask and dried under vacuum with heating and the flask was filled with nitrogen. A portion of the activated $\mathrm{Zn}$ dust (500 mg, $7.65 \mathrm{mmol}, 3$ equiv) was cooled to room temperature. Then, iodo compound $\mathbf{6}$ (842 mg, $2.55 \mathrm{mmol})$ was dissolved in DMF $(10 \mathrm{~mL})$ and added dropwise to the freshly activated $\mathrm{Zn}$ powder under a nitrogen atmosphere and the suspension was stirred at room temperature for $3 \mathrm{~h}$. After completion of $\mathrm{Zn}$ insertion reaction, stirring was stopped and the solid was allowed to settle down. The supernatant was carefully transferred to a suspension of triiodo derivative $9(500 \mathrm{mg}, 0.73 \mathrm{mmol})$ in DMF $(10 \mathrm{~mL})$ at room temperature. Five mol \% tetrakis(triphenylphosphane)palladium $\left(\mathrm{Pd}\left(\mathrm{PPh}_{3}\right)_{4}\right)$ was added to this mixture under inert atmosphere and the reaction mixture stirred at $80{ }^{\circ} \mathrm{C}$ for $12 \mathrm{~h}$. The reaction mixture was cooled to room temperature and washed with water, brine $(3 \times 15 \mathrm{~mL}), 1 \mathrm{M}$ aq $\mathrm{Na}_{2} \mathrm{~S}_{2} \mathrm{O}_{3}$ solution and extracted with EtOAc $(3 \times 10 \mathrm{~mL})$. The combined organic layers were dried over $\mathrm{Na}_{2} \mathrm{SO}_{4}$ and concentrated at reduced pressure. The crude was purified by silica gel column chromatography ( $30 \%$ ethyl acetate/petroleum ether) to afford the Negishi coupling product $10(458 \mathrm{mg}, 68 \%)$ as a colorless solid. $R_{\mathrm{f}}=0.73\left(3: 7\right.$ ethyl acetate/petroleum ether), $[\alpha]_{\mathrm{D}}{ }^{25}+7.78$ (c 1.0, $\left.\mathrm{CHCl}_{3}\right) ;{ }^{1} \mathrm{H} \mathrm{NMR}\left(400 \mathrm{MHz}, \mathrm{CDCl}_{3}\right) \delta 7.75(\mathrm{~s}, 3 \mathrm{H})$, $7.64(\mathrm{~d}, J=8.0 \mathrm{~Hz}, 6 \mathrm{H}), 7.28$ (d, $J=8.0 \mathrm{~Hz}, 6 \mathrm{H}), 5.14$ (d, $J=8.0 \mathrm{~Hz}, 3 \mathrm{H}), 4.67(\mathrm{~d}, J=6.8 \mathrm{~Hz}, 3 \mathrm{H}), 3.77(\mathrm{~s}, 9 \mathrm{H})$, $3.24-3.11(\mathrm{~m}, 6 \mathrm{H}), 1.45(\mathrm{~s}, 27 \mathrm{H}) \mathrm{ppm} ;{ }^{13} \mathrm{C} \mathrm{NMR}(100 \mathrm{MHz}$, $\left.\mathrm{CDCl}_{3}\right) \delta 172.4,155.2,141.9,139.8,135.5,129.9,127.4$, 124.9, 80.0, 54.5, 52.3, 38.0, 28.3 ppm; HRMS-ESI (Q-Tof, $m / z):[\mathrm{M}+\mathrm{Na}]^{+}$calcd for $\mathrm{C}_{51} \mathrm{H}_{63} \mathrm{~N}_{3} \mathrm{NaO}_{12}, 932.4304$; found, 932.4302; IR (neat) $\widetilde{v}_{\max }: 3661,2349,1716,1495,1163,1044$, $755 \mathrm{~cm}^{-1}$.

\section{General procedure for the mono- and dipeptide products 11,12 and 13}

Negishi coupling product $\mathbf{1 0}$ was dissolved in dichloromethane/ trifluoroacetic acid $\left(\mathrm{CH}_{2} \mathrm{Cl}_{2} / \mathrm{TFA}\right.$ 1:1) and the reaction mixture was stirred at room temperature for $1 \mathrm{~h}$. Then, the mixture was concentrated at reduced pressure to remove the solvent and dried under vacuum. Later, without further purification the Negishi coupling deprotection product was reacted with 3 equiv of thiophene 2-carboxylic acid or amino acids ( $N$-Boc-L-valine or Boc-Phe-OH) in the presence of $\mathrm{N}, \mathrm{N}$-diisopropylethylamine (DIPEA, 4 equiv), 2-(1H-benzotriazol-1-yl)-1,1,3,3-tetramethyluronium hexafluorophosphate (HBTU, 9 equiv) in $\mathrm{CH}_{2} \mathrm{Cl}_{2}$. Afterwards, the reaction mixture was stirred at room temperature for $5 \mathrm{~h}$ under an inert atmosphere. After completion of the reaction, the mixture was washed with water, brine $(3 \times 10 \mathrm{~mL})$ and extracted with $\mathrm{CH}_{2} \mathrm{Cl}_{2}(2 \times 10 \mathrm{~mL})$. The combined organic layer was dried over $\mathrm{Na}_{2} \mathrm{SO}_{4}$ and concentrated at reduced pressure. The crude product was purified by silica gel column chromatography $(80 \%$ ethyl acetate/petroleum ether) to afford the $C_{3}$-symmetric mono- and dipeptide derivatives 11,12 and 13 , respectively.

\section{Peptide derivative 11}

Colorless solid; yield $86 \%$ ( $89 \mathrm{mg}$, starting from $100 \mathrm{mg}$ of $\mathbf{1 0}$ ); $R_{\mathrm{f}}=0.46$ (7:3 ethyl acetate/petroleum ether); mp $156-158^{\circ} \mathrm{C}$; $[\alpha]_{\mathrm{D}}{ }^{25}+25.07\left(c 1.0, \mathrm{CHCl}_{3}\right) ;{ }^{1} \mathrm{H}$ NMR $\left(500 \mathrm{MHz}, \mathrm{CDCl}_{3}\right)$ $\delta 7.71(\mathrm{~s}, 3 \mathrm{H}), 7.60(\mathrm{~d}, J=8.0 \mathrm{~Hz}, 6 \mathrm{H}), 7.48(\mathrm{q}, J=3.5 \mathrm{~Hz}$, $6 \mathrm{H}), 7.24(\mathrm{~d}, J=8.0 \mathrm{~Hz}, 6 \mathrm{H}), 7.04(\mathrm{t}, J=4.5 \mathrm{~Hz}, 3 \mathrm{H}), 6.64(\mathrm{~d}$, $J=7.5 \mathrm{~Hz}, 3 \mathrm{H}), 5.10(\mathrm{q}, J=5.5 \mathrm{~Hz}, 3 \mathrm{H}), 3.79(\mathrm{~s}, 9 \mathrm{H})$, 3.35-3.24 (m, 6H) ppm; $\left.{ }^{13} \mathrm{C} \mathrm{NMR} \mathrm{(125} \mathrm{MHz,} \mathrm{CDCl}_{3}\right) \delta 172.0$, $161.5,141.9,139.9,138.2,135.3,130.6,129.9,128.7,127.8$, 127.6, 125.0, 53.6, 52.6, 37.7 ppm; HRMS-ESI (Q-Tof, $\mathrm{m} / \mathrm{z}$ ): $[\mathrm{M}+\mathrm{H}]^{+}$calcd for $\mathrm{C}_{51} \mathrm{H}_{46} \mathrm{~N}_{3} \mathrm{O}_{9} \mathrm{~S}_{3}, 940.2391$; found, 940.2392; IR (neat) $\widetilde{v}_{\max }: 3769,3327,2932,1664,1169,759 \mathrm{~cm}^{-1}$.

\section{Dipeptide 12}

Colorless solid; yield 73\% (97 mg, starting from $100 \mathrm{mg}$ of $\mathbf{1 0}$ ); $R_{\mathrm{f}}=0.59$ (6:4 ethyl acetate/petroleum ether); $\mathrm{mp}<230{ }^{\circ} \mathrm{C}(\mathrm{dec})$; $[\alpha]_{\mathrm{D}}{ }^{25}+20.58\left(c 1.0, \mathrm{CHCl}_{3}\right) ;{ }^{1} \mathrm{H} \mathrm{NMR}\left(400 \mathrm{MHz}, \mathrm{CDCl}_{3}\right)$ $\delta 7.69(\mathrm{~s}, 3 \mathrm{H}), 7.59(\mathrm{~d}, J=8.0 \mathrm{~Hz}, 6 \mathrm{H}), 7.21(\mathrm{~d}, J=7.6 \mathrm{~Hz}$, $6 \mathrm{H}), 6.56(\mathrm{~d}, J=6.8 \mathrm{~Hz}, 3 \mathrm{H}), 5.12(\mathrm{~d}, J=7.2 \mathrm{~Hz}, 3 \mathrm{H}), 4.91(\mathrm{~d}$, $J=6.4 \mathrm{~Hz}, 3 \mathrm{H}), 3.95(\mathrm{~s}, 3 \mathrm{H}), 3.72(\mathrm{~s}, 9 \mathrm{H}), 3.16(\mathrm{~s}, 6 \mathrm{H}), 2.09(\mathrm{~d}$, $J=6.0 \mathrm{~Hz}, 3 \mathrm{H}), 1.41(\mathrm{~s}, 27 \mathrm{H}), 0.92(\mathrm{~d}, J=6.8 \mathrm{~Hz}, 9 \mathrm{H}), 0.87$ $(\mathrm{d}, J=4.0 \mathrm{~Hz}, 9 \mathrm{H}) \mathrm{ppm} ;{ }^{13} \mathrm{C} \mathrm{NMR}\left(100 \mathrm{MHz}, \mathrm{CDCl}_{3}\right) \delta 171.8$, $171.5,155.9,141.9,139.9,135.3,129.8,127.5,124.9,79.9$, 60.0, 53.2, 52.4, 37.8, 31.0, 28.4, 19.3, 17.8 ppm; HRMS-ESI (Q-Tof, $m / z$ ): $[\mathrm{M}+\mathrm{Na}]^{+}$calcd for $\mathrm{C}_{66} \mathrm{H}_{90} \mathrm{~N}_{6} \mathrm{NaO}_{15}, 1229.6356$; found, 1229.6359; IR (neat) $\widetilde{v}_{\text {max }}: 3342,2938,2332,1742,1635$, $1534,1213,754 \mathrm{~cm}^{-1}$.

\section{Dipeptide 13}

Colorless solid; yield $81 \%$ (96 mg, starting from $80 \mathrm{mg}$ of $\mathbf{1 0}$ ); $R_{\mathrm{f}}=0.73$ (6:4 ethyl acetate/petroleum ether); $\mathrm{mp} 204-206{ }^{\circ} \mathrm{C}$; ${ }^{1} \mathrm{H}$ NMR $\left(500 \mathrm{MHz}, \mathrm{CDCl}_{3}\right) \delta 7.70(\mathrm{~s}, 3 \mathrm{H}), 7.56(\mathrm{~d}, J=8.0 \mathrm{~Hz}$, $6 \mathrm{H}), 7.27(\mathrm{~d}, J=7.6 \mathrm{~Hz}, 6 \mathrm{H}), 7.20(\mathrm{t}, J=5.6 \mathrm{~Hz}, 9 \mathrm{H}), 7.10(\mathrm{~d}$, $J=8.0 \mathrm{~Hz}, 6 \mathrm{H}), 6.35(\mathrm{~d}, J=6.80 \mathrm{~Hz}, 3 \mathrm{H}), 4.98(\mathrm{br}, 3 \mathrm{H}), 4.83$ $(\mathrm{d}, J=6.0 \mathrm{~Hz}, 3 \mathrm{H}), 4.35(\mathrm{~d}, J=5.20 \mathrm{~Hz}, 3 \mathrm{H}), 3.70(\mathrm{~s}, 9 \mathrm{H})$, 3.10-3.00 (m, 12H), $1.34(\mathrm{~s}, 27 \mathrm{H}) \mathrm{ppm} ;{ }^{13} \mathrm{C} \mathrm{NMR} \mathrm{(100} \mathrm{MHz,}$ $\left.\mathrm{CDCl}_{3}\right) \delta 171.5,171.0,155.4,142.0,140.0,136.6,135.2$, $129.9,129.5,128.8,127.6,127.1,125.0,80.4,55.9,53.5,52.5$, 38.5, 37.8, 28.4 ppm; HRMS-ESI (Q-Tof, $m / z$ ): $[\mathrm{M}+\mathrm{Na}]^{+}$ calcd for $\mathrm{C}_{78} \mathrm{H}_{90} \mathrm{~N}_{6} \mathrm{NaO}_{15}, 1373.6356$; found, 1373.6359; IR (neat) $\widetilde{v}_{\max }: 3738,3644,2919,2850,2343,1666,1517,814$, $751 \mathrm{~cm}^{-1}$.

\section{Trisamine derivative 14}

Compound 12 (95 mg, $0.07 \mathrm{mmol}$ ) was dissolved in $\mathrm{CH}_{2} \mathrm{Cl}_{2}$ / TFA 1:1 and this mixture was stirred at room temperature for $1 \mathrm{~h}$. At the conclusion of the reaction (TLC monitoring), the 
reaction mixture was concentrated at reduced pressure and dried under vacuum. The crude product was purified by silica gel column chromatography $\left(2 \% \mathrm{MeOH} / \mathrm{CHCl}_{3}\right)$ to obtain the Bocdeprotection product $\mathbf{1 4}(68 \mathrm{mg}, 95 \%)$ as a colorless solid. $R_{\mathrm{f}}=0.46(0.5: 9.5$ methanol/chloroform $) ; \mathrm{mp}:<250{ }^{\circ} \mathrm{C}(\mathrm{dec})$; $[\alpha]_{\mathrm{D}}{ }^{24}+0.97\left(c 1.0, \mathrm{CHCl}_{3}\right) ;{ }^{1} \mathrm{H}$ NMR $\left(500 \mathrm{MHz}, \mathrm{CDCl}_{3}\right)$ $\delta 7.75(\mathrm{~s}, 3 \mathrm{H}), 7.67(\mathrm{~d}, J=7.5 \mathrm{~Hz}, 6 \mathrm{H}), 7.39(\mathrm{~d}, J=8.0 \mathrm{~Hz}$, $6 \mathrm{H}), 3.75(\mathrm{~d}, J=4.5 \mathrm{~Hz}, 3 \mathrm{H}), 3.71(\mathrm{~s}, 9 \mathrm{H}), 3.28-3.24(\mathrm{~m}, 3 \mathrm{H})$, 3.13-3.09 (m, 3H), 2.25 (q, $J=6.0 \mathrm{~Hz}, 3 \mathrm{H}), 1.27$ (s, 3H), 1.08 $(\mathrm{d}, J=6.5 \mathrm{~Hz}, 9 \mathrm{H}), 1.04$ (d, $J=7.0 \mathrm{~Hz}, 9 \mathrm{H}) \mathrm{ppm} ;{ }^{13} \mathrm{C} \mathrm{NMR}$ $\left(125 \mathrm{MHz}, \mathrm{CDCl}_{3}\right) \delta 173.5,170.4,143.9,141.5,138.1,131.4$, $129.0,126.1,60.0,56.1,38.4,32.2,19.4,18.1 \mathrm{ppm}$; HRMS-ESI (Q-Tof $m / z)$ : $[\mathrm{M}+\mathrm{H}]^{+}$calcd for $\mathrm{C}_{51} \mathrm{H}_{67} \mathrm{~N}_{6} \mathrm{O}_{9}$, 907.4964; found, 907.4963; IR (neat) $\widetilde{v}_{\max }: 3779,3240,2928$, $1606,1596,1434,783,505 \mathrm{~cm}^{-1}$.

\section{Supporting Information}

$$
\begin{aligned}
& \text { Supporting Information File } 1 \\
& \text { Copies of }{ }^{1} \mathrm{H},{ }^{13} \mathrm{C} \text { NMR and HRMS spectra of new } \\
& \text { compounds. } \\
& \text { [https://www.beilstein-journals.org/bjoc/content/ } \\
& \text { supplementary/1860-5397-15-33-S1.pdf] }
\end{aligned}
$$

\section{Acknowledgements}

We thank the Department of Science and Technology (DST), New Delhi, India, for financial support and IIT Bombay, for recording spectral data. S.K. thanks the Department of Science and Technology for the award of a J. C. Bose fellowship (SR/ S2/JCB-33/2010), Praj industries, Pune, for Pramod Chaudhari, Chair Professor (Green Chemistry) and CSIR (02(0272)/16/ EMR-II). S.T. thanks the IIT Bombay for the award of a research fellowship.

\section{ORCID ${ }^{\circledR}$ iDs}

Saidulu Todeti - http://orcid.org/0000-0001-5244-7558

\section{References}

1. Dangel, B.; Clarke, M.; Haley, J.; Sames, D.; Polt, R. J. Am. Chem. Soc. 1997, 119, 10865-10866. doi:10.1021/ja972135j

2. Etayo, P.; Ayats, C.; Pericàs, M. A. Chem. Commun. 2016, 52, 1997-2010. doi:10.1039/c5cc08961a

3. Klajnert, B.; Bryszewska, M. Acta Biochim. Pol. 2001, 48, 199-208.

4. Romagnoli, B.; Hayes, W. J. Mater. Chem. 2002, 12, 767-799. doi:10.1039/b110218b

5. Tansey, M. G.; Szymkowski, D. E. Drug Discovery Today 2009, 14, 1082-1088. doi:10.1016/j.drudis.2009.10.002

6. Liu, J.; Bartesaghi, A.; Borgnia, M. J.; Sapiro, G.; Subramaniam, S. Nature 2008, 455, 109-113. doi:10.1038/nature07159

7. Zhu, P.; Liu, J.; Bess, J., Jr.; Chertova, E.; Lifson, J. D.; Grisé, H.; Ofek, G. A.; Taylor, K. A.; Roux, K. H. Nature 2006, 441, 847-852. doi:10.1038/nature 04817
8. Berthelmann, A.; Lach, J.; Gräwert, M. A.; Groll, M.; Eichler, J. Org. Biomol. Chem. 2014, 12, 2606-2614. doi:10.1039/c3ob42251h

9. Kotha, S.; Brahmachary, E. J. Org. Chem. 2000, 65, 1359-1365. doi:10.1021/jo991387v

10. Kotha, S.; Sreenivasachary, N.; Mohanraja, K.; Durani, S. Bioorg. Med. Chem. Lett. 2001, 11, 1421-1423. doi:10.1016/s0960-894x(01)00227-x

11. Kotha, S. Acc. Chem. Res. 2003, 36, 342-351. doi:10.1021/ar020147q 12. Balaram, P. Curr. Opin. Struct. Biol. 1992, 2, 845-851. doi:10.1016/0959-440x(92)90110-s

13. Casabona, D.; Cativiela, C. Synthesis 2006, 2440-2443. doi:10.1055/s-2006-942458

14. Kotha, S.; Halder, S. Synlett 2010, 337-354. doi:10.1055/s-0029-1219149

15. Kotha, S.; Ghosh, A. K. Tetrahedron 2004, 60, 10833-10841. doi:10.1016/j.tet.2004.09.051

16. Kotha, S.; Todeti, S.; Das, T.; Datta, A. Tetrahedron Lett. 2018, 59, 1023-1027. doi:10.1016/j.tetlet.2018.01.084

17. Dash, B. P.; Satapathy, R.; Maguire, J. A.; Hosmane, N. S. Org. Lett. 2008, 10, 2247-2250. doi:10.1021/ol8005248

18. Kashiki, T.; Kohara, M.; Osaka, I.; Miyazaki, E.; Takimiya, K. J. Org. Chem. 2011, 76, 4061-4070. doi:10.1021/jo2005044

19. Mbyas Saroukou, M. S.; Skalski, T.; Skene, W. G.; Lubell, W. D. Tetrahedron 2014, 70, 450-458. doi:10.1016/j.tet.2013.11.043

20. Dash, J.; Trawny, D.; Rabe, J. P.; Reissig, H.-U. Synlett 2015, 26, 1486-1489. doi:10.1055/s-0034-1380716

21. Preis, E.; Dong, W.; Brunklaus, G.; Scherf, U. J. Mater. Chem. C 2015, 3, 1582-1587. doi:10.1039/c4tc02664k

22. Shah, S. R.; Thakore, R. R.; Vyas, T. A.; Sridhar, B. Synlett 2016, 27, 294-300. doi:10.1055/s-0035-1560576

23. Kotha, S.; Todeti, S.; Gopal, M. B.; Datta, A. ACS Omega 2017, 2, 6291-6297. doi:10.1021/acsomega.7b00941

24. Kotha, S.; Todeti, S.; Das, T.; Datta, A. ChemistrySelect 2018, 3, 136-141. doi:10.1002/slct.201702675

25. Kotha, S.; Chakraborty, K.; Brahmachary, E. Synlett 1999, 1621-1623. doi:10.1055/s-1999-2895

26. Thallapally, P. K.; Chakraborty, K.; Carrell, H. L.; Kotha, S.; Desiraju, G. R. Tetrahedron 2000, 56, 6721-6728. doi:10.1016/s0040-4020(00)00493-2

27. El-Bendary, M.; Priest, F. G.; Charles, J.-F.; Mitchell, W. J. FEMS Microbiol. Lett. 2005, 252, 51-56. doi:10.1016/j.femsle.2005.08.027

28. Mitchell, W. J.; Kopidakis, N.; Rumbles, G.; Ginley, D. S.; Shaheen, S. E. J. Mater. Chem. 2005, 15, 4518-4528. doi:10.1039/b508683c

29. Belton, C. R.; Kanibolotsky, A. L.; Kirkpatrick, J.; Orofino, C.; Elmasly, S. E. T.; Stavrinou, P. N.; Skabara, P. J.; Bradley, D. D. C. Adv. Funct. Mater. 2013, 23, 2792-2804. doi:10.1002/adfm.201202644

30. Lai, W.-Y.; He, Q.-Y.; Zhu, R.; Chen, Q.-Q.; Huang, W. Adv. Funct. Mater. 2008, 18, 265-276. doi:10.1002/adfm.200700224

31. Hoang, M. H.; Cho, M. J.; Kim, D. C.; Kim, K. H.; Shin, J. W.; Cho, M. Y.; Joo, J.-s.; Choi, D. H. Org. Electron. 2009, 10, 607-617. doi:10.1016/j.orgel.2009.02.021

32. Ponomarenko, S. A.; Kirchmeyer, S.; Elschner, A.; Huisman, B.-H.; Karbach, A.; Drechsler, D. Adv. Funct. Mater. 2003, 13, 591-596. doi:10.1002/adfm.200304363

33. Kinoshita, M.; Shirota, Y. Chem. Lett. 2001, 30, 614-615. doi:10.1246/cl.2001.614

34. Kotha, S.; Shah, V. R. Amino Acids 2008, 35, 83-88. doi:10.1007/s00726-007-0626-9 
35. Pieters, R. J.; Cuntze, J.; Bonnet, M.; Diederich, F.

J. Chem. Soc., Perkin Trans. 2 1997, 1891-1900.

doi:10.1039/a702627g

36. Gutiérrez-Abad, R.; Illa, O.; Ortuño, R. M. Org. Lett. 2010, 12, 3148-3151. doi:10.1021/ol1010664

37. King, A. O.; Okukado, N.; Negishi, E.-i. J. Chem. Soc., Chem. Commun. 1977, 683-684. doi:10.1039/c39770000683

38. Brittain, W. D. G.; Cobb, S. L. Org. Biomol. Chem. 2018, 16, 10-20. doi:10.1039/c7ob02682j

39. Oswald, C. L.; Carrillo-Márquez, T.; Caggiano, L.; Jackson, R. F. W. Tetrahedron 2008, 64, 681-687. doi:10.1016/j.tet.2007.11.031

40. Rilatt, I.; Caggiano, L.; Jackson, R. F. W. Synlett 2005, 2701-2719. doi:10.1055/s-2005-918950

41. de Loos, M.; van Esch, J. H.; Kellogg, R. M.; Feringa, B. L. Tetrahedron 2007, 63, 7285-7301. doi:10.1016/j.tet.2007.02.066

42. Danner, P.; Morkunas, M.; Maier, M. E. Org. Lett. 2013, 15 , 2474-2477. doi:10.1021/ol4009409

43. Bender, A. M.; Griggs, N. W.; Gao, C.; Trask, T. J.; Traynor, J. R.; Mosberg, H. I. ACS Med. Chem. Lett. 2015, 6, 1199-1203. doi:10.1021/acsmedchemlett.5b00344

44. Li, Z.; Ke, F.; Deng, H.; Xu, H.; Xiang, H.; Zhou, X. Org. Biomol. Chem. 2013, 11, 2943-2946. doi:10.1039/c3ob40464a

45. Rajwar, D.; Sun, X.; Cho, S. J.; Grimsdale, A. C.; Fichou, D. CrystEngComm 2012, 14, 5182-5187. doi:10.1039/c2ce25530h 46. Zhao, S.; Kang, L.; Ge, H.; Yang, F.; Wang, C.; Li, C.; Wang, Q.; Zhao, M. Synth. Commun. 2012, 42, 3569-3578. doi:10.1080/00397911.2011.585731

\section{License and Terms}

This is an Open Access article under the terms of the Creative Commons Attribution License (http://creativecommons.org/licenses/by/4.0). Please note that the reuse, redistribution and reproduction in particular requires that the authors and source are credited.

The license is subject to the Beilstein Journal of Organic Chemistry terms and conditions:

(https://www.beilstein-journals.org/bjoc)

The definitive version of this article is the electronic one which can be found at: doi:10.3762/bjoc. 15.33 